\title{
The Prevalence and Impact of Overlapping Rome IV-Diagnosed Functional Gastrointestinal Disorders on Somatization, Quality of Life, and Healthcare Utilization: A Cross-Sectional General Population Study in Three Countries
}

Imran Aziz, MBChB, MD르. Olafur S. Palsson, PsyD², Hans Törnblom, MD, PhD¹, Ami D. Sperber, MD, MSPH ${ }^{3}$, William E. Whitehead, $\mathrm{PhD}^{2}$ and Magnus Simrén, MD, PhD ${ }^{1,2}$

OBJECTIVES: The population prevalence of Rome IV-diagnosed functional gastrointestinal disorders (FGIDs) and their cumulative effect on health impairment is unknown.

METHODS: $\quad$ An internet-based cross-sectional health survey was completed by 5,931 of 6,300 general population adults from three English-speaking countries (2100 each from USA, Canada, and UK). Quota-based sampling was used to generate demographically balanced and population representative samples with regards to age, sex, and education level. The survey enquired for demographics, medication, surgical history, somatization, quality of life (QOL), doctor-diagnosed organic GI disease, and criteria for the Rome IV FGIDs. Comparisons were made between those with Rome IV-diagnosed FGIDs against non-GI (healthy) and organic GI disease controls.

RESULTS: $\quad$ The number of subjects having symptoms compatible with a FGID was 2,083 (35\%) compared with $3,421(57.7 \%)$ non-GI and $427(7.2 \%)$ organic GI disease controls. The most frequently met diagnostic criteria for FGIDs was bowel disorders ( $n=1,665,28.1 \%$ ), followed by gastroduodenal ( $n=627,10.6 \%$ ), anorectal $(n=440,7.4 \%)$, esophageal $(n=414,7 \%)$, and gallbladder disorders $(n=10,0.2 \%)$. On average, the 2,083 individuals who met FGID criteria qualified for 1.5 FGID diagnoses, and 742 of them (36\%) qualified for FGID diagnoses in more than one anatomic region. The presence of FGIDs in multiple regions was associated with increasing somatization, worse mental/physical QOL, more medical therapies, and a higher prevalence of abdominal surgeries; all $P<0.001$. Notably, individuals with FGIDs in multiple regions had greater somatization and worse QOL than organic GI disease controls.

CONCLUSIONS: Roughly a third of the general adult population fulfils diagnostic criteria for a Rome IV FGID. In a third of this subset multiple GI regions are involved and this overlap is associated with increased health impairment.

SUPPLEMENTARY MATERIAL is linked to the online version of the paper at http://www. nature.com/ajg

Am J Gastroenterol 2018; 113:86-96; doi:10.1038/ajg.2017.421; published online 14 November 2017

\section{INTRODUCTION}

The Rome IV consensus documents have recently been published to help in the understanding, diagnostic processing, and management of functional gastrointestinal disorders (FGIDs) $(1,2)$. They define FGIDs as disturbances of the brain-gut axis in the absence of organic pathology. Furthermore, in adults the

${ }^{1}$ Department of Internal Medicine and Clinical Nutrition, Institute of Medicine, Sahlgrenska Academy, University of Gothenburg, Gothenburg, Sweden; ${ }^{2}$ Center for Functional Gastrointestinal and Motility Disorders, University of North Carolina, Chapel Hill, North Carolina, USA; ${ }^{3}$ Faculty of Health Sciences, Ben-Gurion University of the Negev, Beer-Sheva, Israel. Correspondence: Imran Aziz, MBChB, MD, Department of Internal Medicine and Clinical Nutrition, Institute of Medicine, Sahlgrenska Academy, University of Gothenburg, 41345 Gothenburg, Sweden. E-mail: imran.aziz@sth.nhs.uk

Received 27 June 2017; accepted 3 October 2017 
FGIDs now comprise 33 distinct clinical entities which can be grouped into one of six anatomical or organ domains: esophageal, gastroduodenal, gallbladder, bowel, anorectal, and centrally mediated disorders of GI pain $(1,2)$.

FGIDs are common and account for the majority of GI consultations seen in primary and secondary-care (3-17). They represent a public health burden given their chronic remitting-relapsing course, which significantly impacts on an individual's general state of well-being aside from their specific GI complaint $(3,4)$. For example, previous studies have shown FGIDs to be associated with psychological disturbances, somatization, poor quality of life (QOL), and increased healthcare interventions such as surgery (18-34). Moreover, several recent studies have shown that FGIDs commonly overlap and are associated with increased extraintestinal symptom burden and physician consultations (35-38). However, research on the prevalence of FGIDs and their impact on health impairment has been limited, both in the general population and in secondary care settings, to evaluations within single communities or purely focusing on distinct clinical entities such as irritable bowel syndrome (IBS), functional dyspepsia, and gastroesophageal reflux (3-38). To our knowledge, there have been no studies evaluating the epidemiology of all FGIDs comprehensively across multiple community populations to assess how the accumulation of FGIDs interacts with measures of general health.

This absence of studies highlights the need for a multi-national study and we addressed this issue by using data from a largescale, cross-sectional, population-based adult survey across three English-speaking countries to help understand the epidemiology and clinical associations of Rome IV-diagnosed FGIDs, while using subjects without GI disorders (healthy) and those with organic GI disease as comparative control groups. We hypothesised that Rome IV-diagnosed FGIDs are common and that the accumulation of overlapping FGIDs would be associated with greater health impairment.

\section{METHODS}

\section{Questionnaire}

The comprehensive study questionnaire consisted of two main sections: (a) the entire Rome IV diagnostic questionnaire for adults that was recently developed and published by the Rome Foundation (39), and (b) an ad hoc supplemental questionnaire designed to address many factors that could be associated with FGIDs including demographics, medication, abdominal surgical history, doctor-diagnosed organic GI disease, somatization, and QOL. These are detailed below.

1. Demographic data-questions enquiring for age, gender, and ethnicity.

2. Medical history-information was sought with regards to healthcare visits and we also asked individuals to indicate on a checklist if they had a doctor diagnosis of any organic GI disease. The checklist comprised peptic ulcer disease, celiac disease, inflammatory bowel disease, diverticulitis, and upper or lower GI cancer. Furthermore, we enquired for weekly intake of medication relevant to GI-related disorders: GI-specific medication (laxatives, anti-diarrheals, antiemetics, antacids, antispasmodics), analgesics (prescribed and over-the-counter), complementary medicine (herbal remedies and traditional Chinese medicine), and psychotropics (anxiolytics and antidepressants). Finally, subjects were asked as to a history of abdominal surgery including cholecystectomy, appendectomy, hysterectomy, bowel resection, or any other abdominal surgery (the details of which were not further elaborated upon).

3. Rome IV diagnostic questionnaire (39) - this validated questionnaire, which presents respondents with 26-86 questions depending on applicable skip patterns, has adequate sensitivity and excellent specificity for the diagnosis of FGIDs, is fully understandable to at least $90 \%$ of adults, and shows good test-retest reliability. It has now been benchmarked as the screening tool for FGIDs, their inclusion into clinical trials, and for performing epidemiological surveys. For the purpose of our study we categorised FGIDs in accordance with the six-region division of these disorders in the Rome diagnostic system, i.e., into esophageal, gastroduodenal, gallbladder, bowel, anorectal, and centrally mediated disorders of GI pain.

4. Patient health questionnaire (PHQ)-12 somatization score $(40,41)$ - the PHQ-12 is a modified version of the widely used PHQ-15 somatization questionnaire that excludes the three GI symptoms (nausea, abdominal pain, altered bowel habit), as these are likely to be directly related to FGIDs. As a result, the PHQ-12 only records bothersome non-GI symptoms over the past month. The 12 symptoms assessed are back pain, limb pain, headaches, chest pain, dizziness, fainting spells, palpitations, breathlessness, menstrual cramps, dyspareunia, insomnia, and lethargy. Subjects were asked to rate how much they had been troubled by these 12 symptoms over the last four weeks as 0 ("not bothered at all"), 1 ("bothered a little"), or 2 ("bothered a lot"). The PHQ-12 responses can be used to calculate the number of sites reporting somatic symptoms and the overall severity of somatic symptoms (PHQ-12 somatization score). Higher scores represent greater somatization.

5. Short form (SF)-8 score (42) - this is a validated questionnaire used in large scale epidemiological studies to assess general health-related QOL over the past month. The eight items enquire about physical functioning, physical role, bodily pain, general health perceptions, vitality, social functioning, emotional role, and mental health. These can then be aggregated to form a physical component score (PCS) and a mental component score, which are normalised to the general population that has a mean score of 50 (42). A high PCS or mental component score represents better QOL, whereas low scores represent poorer QOL.

\section{Questionnaire distribution and completion}

Qualtrics (Provo, UT, USA), a global market survey company, was commissioned in the year 2015 to provide a nationally 
representative general population sample of adults from three English-speaking countries; the USA, Canada, and UK. Based on an estimated prevalence of the major functional GI disorders of $5-10 \%$, a sample size of 2,000 individuals/country was deemed to be adequate for the planned analyses. Th e individuals recruited were from those who had already registered to participate in various surveys, such as opinion polls and health studies. Participants received points from Qualtrics including an opportunity to receive shopping credits; no monetary compensation or gift was made. Quota-based sampling was used to ensure equal proportion of sex (50:50), age groups (40\% aged 19-39, 40\% aged 40-64, and $20 \%$ aged 65 and older), and education level (30\% maximum with $>16$ years of formal education) across the countries. Subjects were invited to complete a "health survey". In order to avoid selection bias, no information was given to indicate that the survey purpose was to examine GI symptoms. All patients read an online consent form and electronically accepted study enrolment, aft er which they completed the survey. Th e median completion time was $19 \mathrm{~min}$ with multiple quality assurance methods built into the survey to minimise bias and poor quality reporting. Th ese included allowing only one response from each computer device and excluding respondents who failed two attention-test questions or were excessively inconsistent on three GI diagnostic questions that were presented twice in the survey specifi cally to test consistency of symptom reporting.

\section{Statistics}

Statistical analysis was carried out using SPSS version 21.0 soft ware (SPSS, Chicago, IL, USA), with signifi cance set at a $P$ value of $<0.05$. Th ere were no missing data because the online questionnaire required participants to complete each applicable question before being allowed to move onto the next step. Categorical variables were summarized by descriptive statistics, including total numbers and percentages, with comparisons between groups performed using the chi-square test. Continuous variables were summarized by mean and standard deviation, with diff erences between multiple independent groups assessed using the one-way analysis of variance. Post hoc pair-wise comparisons were performed using Bonferroni correction.

Our hypothesis that the accumulation of overlapping FGIDs will be associated with worsening ill-health (somatization, QOL, medication use, and surgical interventions) was assessed using analysis of variance with linear contrast analysis to look for a linear trend, with eff ect size demonstrated as partial eta squared $\left(\eta^{2}\right)$; small to medium eff ects 0.047-0.11, and large eff ects $>0.11$ (43). Finally, the risk of having undergone specifi $c$ types of abdominal surgery with accumulating FGIDs was determined using odds ratios (OR) and $95 \%$ confi dence intervals ( $95 \%$ C.I.), with healthy subjects serving as the control reference.

\section{RESULTS}

\section{Survey population characteristics}

Th e survey was completed by 6,300 individuals, of which there were 2,100 from each of the three countries. Th ere were 369
(5.9\%) inconsistent responders who were excluded from analysis, leaving responses from 5,931 individuals in the analysis data set; 1,949 from USA, 1988 from Canada, and 1,994 from UK. As shown in Supplementary Table 1A-C, the geographical distribution of subjects in our sample closely matched the relative population distribution within each country (by state, province/territory, or government region). The only significant exception was underrepresentation of subjects from Quebec in the Canadian sample, owing to the fact that $84 \%$ of Quebec speaks French as their primary language, whereas the survey could only be completed in English. Comparison of age decade distribution of the samples with the national populations of the three countries is shown in Supplementary Table 2A-D. All age decades in the sample had sufficient numbers to represent their respective populations, and the age group of 70 years and older amounted to one-tenth of the total sample with $>500$ individuals in the combined three-country sample.

The characteristics of the 5,931 subjects are shown in Table $\mathbf{1}$. The mean age in the total study sample was 47.4 years, with $49.2 \%$ being female and $72 \%$ of white ethnicity. The use of medication was reported by $48.3 \%$, which comprised analgesics (29.3\%), GIspecific medication (25.5\%), psychotropics (16.7\%), and complementary medicine (7.2\%). A history of abdominal surgery was reported by $25.2 \%$, most commonly appendectomy $(10.2 \%)$, cholecystectomy (8\%), and hysterectomy (6.3\%). The PHQ-12 score in the general population was 4.8 , with the norm-based SF-8 QOL physical and mental component scores recording similarly at $\sim 49$ points.

\section{The prevalence of gastrointestinal diseases}

In $57.7 \%$ ( $n=3421)$ no GI disorder was discernible on the Rome diagnostic questionnaire or reported by the subjects, and these individuals were subsequently classed as non-GI (healthy) controls. As shown in Table 2, a doctor-diagnosis of organic GI disease was reported by $7.2 \%(n=427)$ of the study sample, and those subjects were classed as organic GI controls. Diverticulitis (2.9\%) predominated in this subgroup, followed by peptic ulcer disease $(1.7 \%)$, inflammatory bowel disease (1.4\%), and GI cancer $(1.1 \%)$, with celiac disease $(0.6 \%)$ being the least common. Rome IV symptom criteria for any FGID, and without organic GI disease, were met by $35.1 \%(n=2083)$ of the subjects, and this was similar across the three countries; $P=0.25$. In the entire population sample the functional bowel disorders predominated at $28.1 \%(n=1665)$, followed by gastroduodenal $(10.6 \%, n=627)$, anorectal $(7.4 \%, n=440)$, and esophageal $(7.0 \%, n=414)$ disorders. Gallbladder disorders $(0.2 \%, n=10)$ were rare and only one person fulfilled criteria for a centrally mediated disorder of GI pain $(0.02 \%)$; as a result these two FGID groups were excluded from further analysis.

With regards to differences between the three countries the Canadian cohort were significantly more likely to have peptic ulcer disease compared with the UK. In contrast, functional gastroduodenal disorders were significantly more prevalent in the US cohort $(12.3 \%)$ than in subjects from Canada $(9.8 \%)$ or UK $(9.7 \%)$. The prevalence of functional esophageal disorders was also highest in 
Table 1. The overall characteristics and comparison between FGID subjects against healthy and organic GI disease controls

\begin{tabular}{|c|c|c|c|c|c|}
\hline & $\begin{array}{l}\text { Overall } \\
(n=5931)\end{array}$ & $\begin{array}{c}\text { Healthy/non-GI } \\
(n=3,421 ; 57.7 \%)\end{array}$ & $\begin{array}{c}\text { FGIDs } \\
(n=2,083 ; 35.1 \%)\end{array}$ & $\begin{array}{l}\text { Organic GI disease } \\
(n=427 ; 7.2 \%)\end{array}$ & $P$-value ${ }^{a}$ \\
\hline \multicolumn{6}{|l|}{ Demographics } \\
\hline Female & $2,918(49.2 \%)$ & $1,459(42.6 \%)$ & $1,236(59.3 \%)$ & $223(52.2 \%)$ & $<0.001^{\mathrm{b}, \mathrm{c}, \mathrm{d}}$ \\
\hline White ethnicity & $4,271(72 \%)$ & $2,403(70.2 \%)$ & $1,529(73.4 \%)$ & $339(79.4 \%)$ & $<0.001^{\mathrm{b}, \mathrm{c}, \mathrm{d}}$ \\
\hline More than once yearly healthcare visits & $3,407(57.4 \%)$ & $1,684(49.2 \%)$ & $1,392(66.8 \%)$ & $331(77.5 \%)$ & $<0.001^{\mathrm{b}, \mathrm{c}, \mathrm{d}}$ \\
\hline \multicolumn{6}{|l|}{ Medication taken at least once weekly } \\
\hline GI-specific medication & $1,512(25.5 \%)$ & $476(13.9 \%)$ & $817(39.2 \%)$ & $219(51.3 \%)$ & $<0.001^{\mathrm{b}, \mathrm{c}, \mathrm{d}}$ \\
\hline Analgesics & $1,737(29.3 \%)$ & $669(19.6 \%)$ & $871(41.8 \%)$ & $197(46.1 \%)$ & $<0.001^{\mathrm{b}, \mathrm{c}}$ \\
\hline Psychotropic medicine & $993(16.7 \%)$ & $340(9.9 \%)$ & $535(25.7 \%)$ & $118(27.6 \%)$ & $<0.001^{\mathrm{b}, \mathrm{c}}$ \\
\hline Mean number of medication, $\max =11$ (s.d.) & $1.05(1.5)$ & $0.6(1.0)$ & $1.6(1.7)$ & $2(2)$ & $<0.001^{\mathrm{b}, \mathrm{c}, \mathrm{d}}$ \\
\hline \multicolumn{6}{|l|}{ Previous surgery } \\
\hline Cholecystectomy & $472(8 \%)$ & $196(5.7 \%)$ & $204(9.8 \%)$ & $72(16.9 \%)$ & $<0.001^{\mathrm{b}, \mathrm{c}, \mathrm{d}}$ \\
\hline Hysterectomy & $372(6.3 \%)$ & $136(4 \%)$ & $161(7.7 \%)$ & $75(17.6 \%)$ & $<0.001^{\mathrm{b}, \mathrm{c}, \mathrm{d}}$ \\
\hline Appendectomy & $603(10.2 \%)$ & $295(8.6 \%)$ & $218(10.5 \%)$ & $90(21.1 \%)$ & $<0.001^{\mathrm{c}, \mathrm{d}}$ \\
\hline Bowel resection & $108(1.8 \%)$ & $24(0.7 \%)$ & $16(0.8 \%)$ & $68(15.9 \%)$ & $<0.001^{\mathrm{c}, \mathrm{d}}$ \\
\hline Other abdominal or pelvic surgery & $460(7.8 \%)$ & $176(5.1 \%)$ & $216(10.4 \%)$ & $68(15.9 \%)$ & $<0.001^{\mathrm{b}, \mathrm{c}, \mathrm{d}}$ \\
\hline Any of the above abdominal surgeries & $1492(25.2 \%)$ & $659(19.3 \%)$ & $606(29.1 \%)$ & $227(53.2 \%)$ & $<0.001^{\mathrm{b}, \mathrm{c}, \mathrm{d}}$ \\
\hline Mean number of abdominal surgeries, $\max =5$ (s.d.) & $0.34(0.7)$ & $0.24(0.55)$ & $0.39(0.7)$ & $0.9(1)$ & $<0.001^{b, c, d}$ \\
\hline Mental component score, MCS & $49(10.9)$ & $52.1(8.8)$ & $44.1(12.1)$ & $47.9(11.3)$ & $<0.001^{\mathrm{b}, \mathrm{c}, \mathrm{d}}$ \\
\hline
\end{tabular}

FGIDs, functional gastrointestinal disorders; GI, gastrointestinal; PCS, physical component score; PHQ, patient health questionnaire; MCS, mental component score.

Note: Superscript letters are significantly different at a $P<0.05$.

${ }^{a} P$-value is difference between non-GI, FGIDs and organic GI disease subjects.

'Indicates the FGID group is significantly different compared with healthy group.

Indicates the organic group is significantly different compared with healthy group.

Indicates organic group is significantly different compared with FGID group.

those from the USA (8.4\%), which was non-signifi cant compared with Canada (6.8\%) but signifi cantly greater than the UK (5.8\%). Th e prevalence of functional bowel and a norectal disorders $\mathrm{w}$ as similar across the countries.

\section{Comparison of subjects with FGIDs against non-GI and organic} GI disease controls

Table 1 compares the FGIDs group against organic GI disease and non-GI (healthy) controls. Th e FGIDs group was younger, and more female predominant, whereas those with organic GI diseases were older, compared with the non-GI controls. There was also a significant stepwise increase from healthy controls to FGIDs and organic GI disease subjects with regard to frequency of healthcare visits, and mean number of medical and surgical interventions. However, it was noteworthy that only rates of cholecystectomy and hysterectomy, but not appendectomy or bowel resection, were significantly higher in subjects with FGIDs compared with non-GI controls.

Both FGID and organic GI disease subjects scored significantly worse in somatization and QOL compared with non-GI controls. As there was a higher female prevalence among FGIDs compared to non-GI (healthy) controls, we evaluated whether removing 
Table 2. Prevalence of gastrointestinal disorders within the adult population in the USA, Canada, and UK

\begin{tabular}{|c|c|c|c|c|c|}
\hline & $\begin{array}{c}\text { Overall } \\
n=5931\end{array}$ & $\begin{array}{c}\text { United States } \\
n=1949\end{array}$ & $\begin{array}{l}\text { Canada } \\
n=1988\end{array}$ & $\begin{array}{c}\text { United Kingdom } \\
\quad n=1994\end{array}$ & $P$-value ${ }^{a}$ \\
\hline Organic gastrointestinal disease & $427(7.2 \%)$ & $149(7.6 \%)$ & $155(7.8 \%)$ & $123(6.2 \%)$ & 0.1 \\
\hline Inflammatory bowel disease & $82(1.4 \%)$ & $24(1.2 \%)$ & $33(1.7 \%)$ & $25(1.3 \%)$ & 0.4 \\
\hline Gastrointestinal cancer & $64(1.1 \%)$ & $22(1.1 \%)$ & $21(1.1 \%)$ & $21(1.1 \%)$ & 1.0 \\
\hline Diverticulitis & $171(2.9 \%)$ & $69(3.5 \%)$ & $50(2.5 \%)$ & $52(2.6 \%)$ & 0.1 \\
\hline Functional gastrointestinal disorders & $2083(35.1 \%)$ & $683(35 \%)$ & $724(36.4 \%)$ & $676(33.9 \%)$ & 0.25 \\
\hline Esophageal Disorders & $414(7 \%)$ & $164(8.4 \%)$ & $135(6.8 \%)$ & $115(5.8 \%)$ & $0.01^{c}$ \\
\hline Gastroduodenal disorders & $627(10.6 \%)$ & $240(12.3 \%)$ & $194(9.8 \%)$ & $193(9.7 \%)$ & $0.01^{c, d}$ \\
\hline Gallbladder disorders & $10(0.2 \%)$ & $7(0.4 \%)$ & $2(0.1 \%)$ & $1(0.1 \%)$ & 0.04 \\
\hline Anorectal disorders & $440(7.4 \%)$ & $148(7.6 \%)$ & $150(7.5 \%)$ & $142(7.1 \%)$ & 0.8 \\
\hline \multicolumn{6}{|c|}{$\begin{array}{l}\text { Note: superscript letters are significantly different at a } P<0.05 \text {. } \\
\text { a } P \text {-value is difference between countries. } \\
\text { 'Indicates Canada is significantly different compared with UK. } \\
\text { Indicates the USA is significantly different compared with UK. } \\
\text { Indicates the USA is significantly different compared with Canada. }\end{array}$} \\
\hline
\end{tabular}

menstrual cramps and dyspareunia from the somatisation analysis would aff ect the diff erence seen between the groups, but somatisation still remained signifi cantly greater in subjects with FGIDs.

\section{Presence of FGIDs in multiple GI regions and its effect on health impairment}

I $\mathrm{n}$ the 2,083 subjects meeting Rome criteria for FGIDs, 64\% $(n=1341)$ had one GI region aff ected by a FGID and 36\% $(n=742)$ had more than one aff e cted G I r egion; $24 \%$ ( $n=493)$ h ad t wo aff ected regions, $8 \%(n=166)$ had three, and $4 \%(n=83)$ had symptoms meeting FGID criteria in four GI regions (Figure 1). Th i s gave a total of 3,157 aff ected GI regions in 2083 FGID subjects, averaging 1.5 GI regions per affl icted subject.

Furthermore,Figure 2 depicts in detail the overlapping relationship between the FGIDs. It reveals a complex interaction with multiple overlapping permutations between the FGIDs. In summary, the $64 \%$ of subjects with an isolated FGID reported bowel $(47.8 \%)$, gastroduodenal (9.2\%), esophageal (4.7\%), and anorectal $(2.7 \%)$ disorders. In the remaining 36\% with overlapping FGIDs, the most frequent overlap was between bowel-anorectal (9.1\%), bowelgastroduodenal (7.5\%) and bowel-esophageal (4.2\%). The 1 east frequent overlap was seen between anorectal-esophageal $(0.1 \%)$.

Linear contrast analysis demonstrated that with the increase in overlapping FGIDs there was a signifi cant linear trend asso-ciated with higher PHQ-12 somatization scores, more somatic symptoms, poorer mental and physical SF-8 QOL scores, greater consumption of medical therapies, and a higher prevalence of sur-gical interventions (Figure 3). Th e risk of specifi $c$ types of abdomi-nal surgery with accumulating spread of FGIDs is reported in

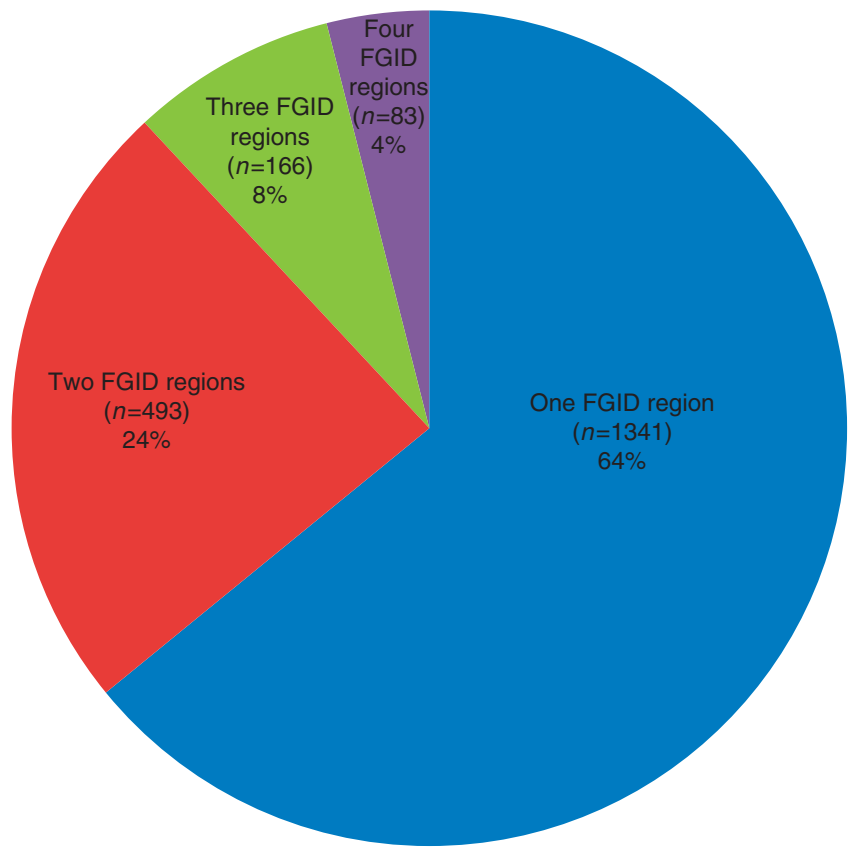

Figure 1. The relationship between number of people with FGIDs and number of affected GI regions. There are 2083 people who fulfilled criteria for FGIDs with the total number of affected $\mathrm{Gl}$ regions being 3157 . The mean ratio of affected GI segments per FGID subject was 1.5 to 1 (3157/2083).

Table 3, showing increasing rates of cholecystectomy and hysterectomy, but not appendectomy or bowel resection. The accumulation of FGID-affected GI regions was also associated with increased frequency of more than once yearly health care visits, ranging from 


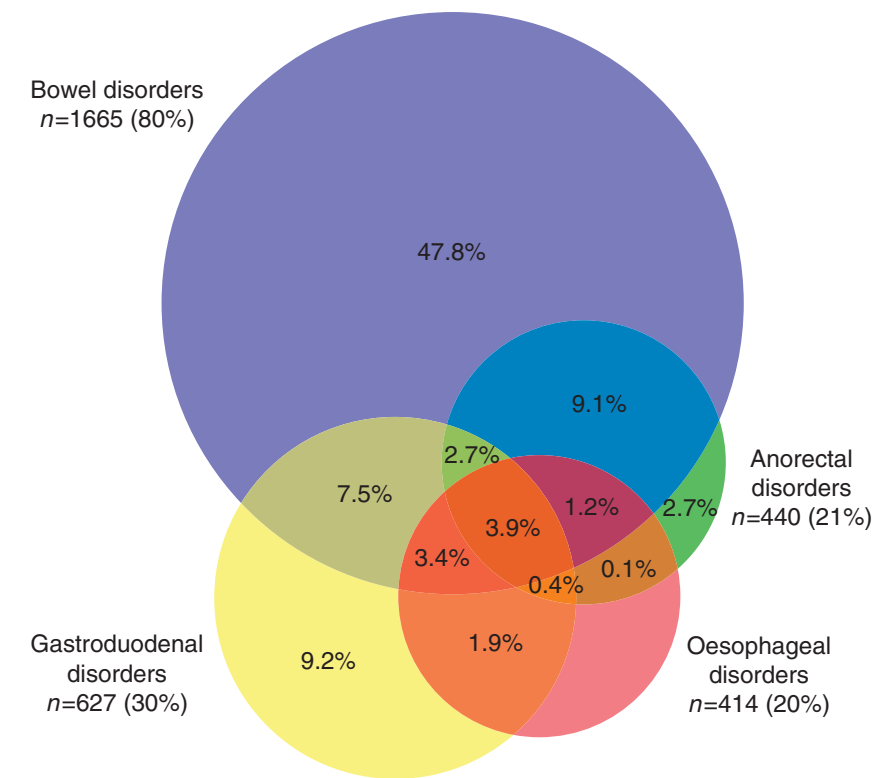

Figure 2. Venn diagram showing the overlap between FGIDs ( $n=2,083)$. Note: (i) Numbers not shown in figure; the overlap between bowel-oesophageal, which was $4.2 \%$, and gastroduodenal-anorectal, which was $0.9 \%$.

(ii) The areas within the diagram are not quite proportional to the numbers. (iii) Data for gallbladder $(n=10)$ and centrally mediated disorders of $\mathrm{Gl}$ pain $(n=1)$ not presented as small numbers.

49.2\% for no FGIDs, $64.4 \%$ for one region with FGID, $68.8 \%$ for two regions, $74.1 \%$ for three regions, and $79.5 \%$ for four regions $(P<0.001)$.

Finally, we also compared the eff e ct of a ccumulating F GIDaff e cted $\mathrm{r}$ egions o $\mathrm{n}$ i ll-health $\mathrm{w}$ ith o rganic $\mathrm{G}$ I d isease $\mathrm{c}$ ontrols. Individuals with FGID in only one region of their GI tract had similar PHQ-12 somatization scores and number of somatic symptoms as seen in organic GI disease subjects $(P=0.56$ and $P=0.94$, respectively), but the presence of $\geq 2$ regions with FGIDs led to higher somatization scores $(P<0.001)$. In terms of mental component score SF8-QOL, the presence of one $(P=0.003)$ or more FGID regions $(P<0.001)$ led to worse scores than organic GI disease subjects. Further, presence of FGID in only one region was associated with better PCS-QOL than organic GI disease subjects $(P=0.003)$, FGID in two regions was associated with similar scores $(P=1.0)$, but subjects with three $(P=0.005)$ and four FGID aff ected regions $(P<0.001)$ had worse PCS-QOL scores than organic GI disease subjects. For medication use, subjects with FGID in one region had less medication use than organic GI diseases $(P<0.001)$, those with two FGID regions had equivalent use $(P=0.9)$, and those with three $(P=0.01)$ and four FGID aff ected regions $(P \varangle 0.001)$ had greater medication use. With regards to surgery, individuals with FGIDs in $\leq 3$ regions had lower overall surgical interventions than organic GI disease subjects $(P<0.001)$ and those with four aff ected regions had similar rates $(P=0.29)$. In summary, other than surgery, the increasing spread of FGIDs across multiple GI regions led to health impairment exceeding that reported by organic GI disease subjects.

\section{DISCUSSION}

Having used the recently published Rome IV diagnostic questionnaire we present the first large-scale m ulti-national s tudy evaluating the total prevalence of FGIDs and their overlap or spread across GI regions in the general adult population. Moreover, we compare FGID subjects with both healthy and organic GI disease controls, whilst also determining the eff ect of overlapping FGIDs on somatization, QOL, and healthcare utilization. Th is has allowed novel insights to be gained into the magnitude of FGIDs and their interaction with general health and wellbeing.

We identified that a third (35\%) of the general a dult population from the USA, Canada, and UK fulfi ls diagnostic criteria for Rome IV FGIDs, with bowel disorders representing the majority of these cases, followed by gastroduodenal, anorectal, and esophageal disorders. In contrast, gallbladder disorders are infrequent and centrally mediated disorders of GI pain exceedingly rare. We have also shown that the FGIDs group (along with organic GI disease subjects) have worse somatization scores, poorer QOL, and excess healthcare utilization compared with healthy controls. Furthermore, we note that in FGID subjects, symptoms qualifying for FGID diagnosis are present in 1.5 GI regions on average; this is owing to more than a third of FGID subjects having multiple affected GI regions, with the most frequent overlaps being co-existence of symptoms of bowel disorders with those of anorectal, gastroduodenal, and esophageal disorders. Moreover, the diagnosis of FGIDs in multiple GI regions was associated with increasing extra-intestinal symptom scores, medication use and surgical intervention which, other than surgery, also exceeded those reported by organic GI disease subjects. Th e se fi ndings are consistent with the recent literature, although studies in this fi eld are limited to community-based studies evaluating the eff ect of few overlapping FGIDs (i.e., IBS/functional dyspepsia/gastroesophageal refl ux) on either somatization, QOL, symptom-burden and consultation behavior, or a secondary care study evaluating multiple FGIDs and showing that their step-wise accumulation leads to increasing anxiety and depression (35-38). Our study augments these fi $\mathrm{n}$ dings by p roviding a n o verarching p erspective of all FGIDs and the eff ect of their overlap on multiple health parameters (including for the fi r st time, s urgery), w hile u sing a control group. As such, our fi n dings have i mportant i mplications in that they highlight the need for future planning of clinical service and research in the fi eld of FGIDs. In the United States, three of the leading six GI diagnoses at ambulatory care visits are accounted for by motility or functional disorders; refl ux symptoms, constipation, and IBS (44). Moreover, FGIDs incur signifi cant costs to patients, healthcare systems (both for GI and non-GI related care) and society through work absenteeism and presenteeism. In 2004, the direct and indirect costs for refl ux symptoms and all other FGIDs combined amounted to $\$ 16$ billion in the USA, which was almost a tenth of the total expenditure for all digestive diseases (44). Factors that are associated with healthcare seeking behavior in FGIDs include poor QOL and psychological symptoms (45). Hence, our results indicating a signifi cant adverse impact of the spread of FGIDs to multiple GI regions indicates 

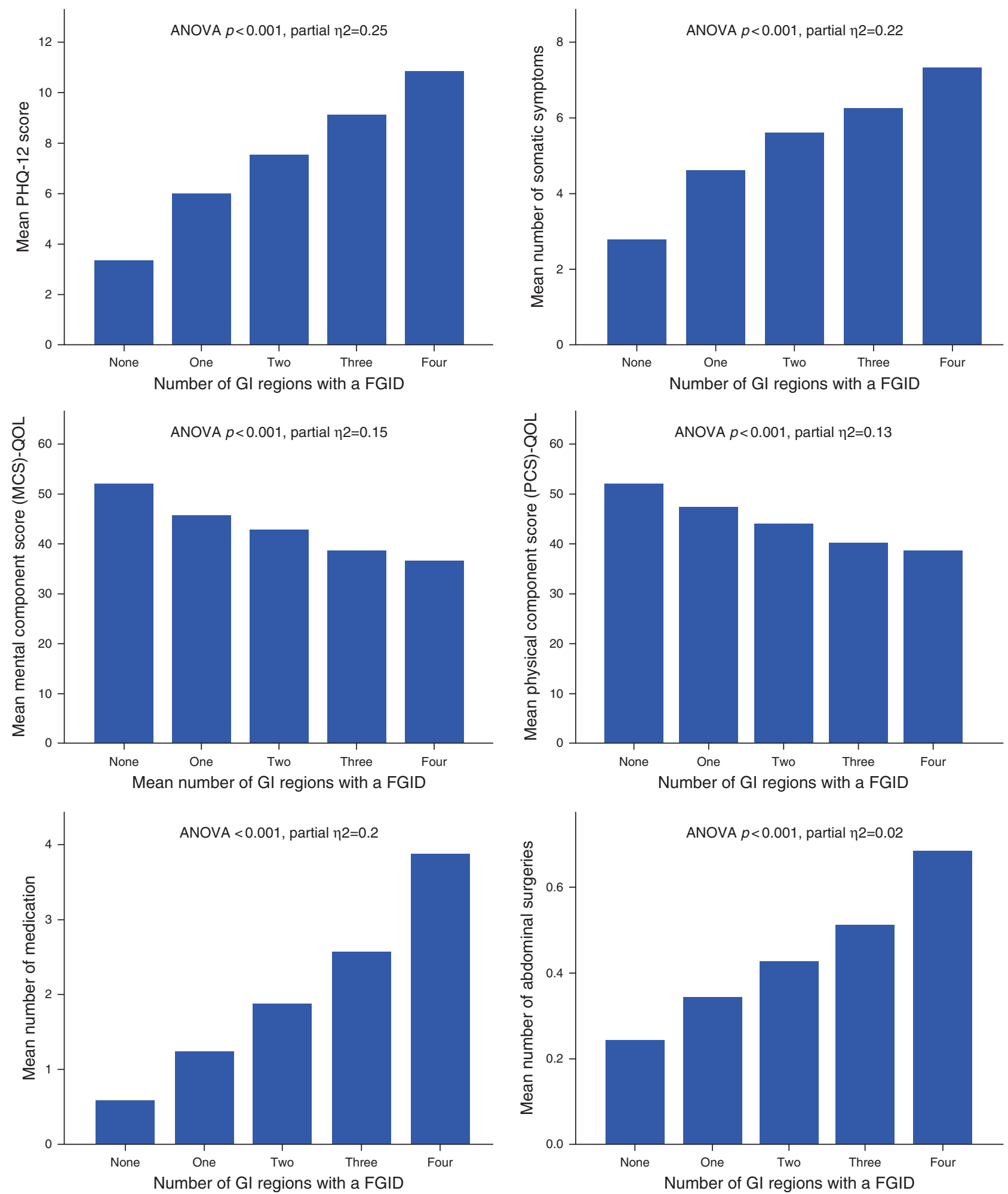

Figure 3. Linear relationship between number of GI regions affected with FGIDs and somatization, quality of life, medication use, and surgeries.

that individuals suff ering from such broad affl iction can be expected to have greater medical care needs and perhaps diff er-ent treatment requirements compared with other FGID patients. We therefore suggest future clinical and research studies clearly distinguish between isolated and multi-region FGIDs, to help toward the understanding of pathophysiological mechanisms and the optimization of treatment. In view of the widespread symptomatology incurred by subjects with overlapping FGIDS it may 


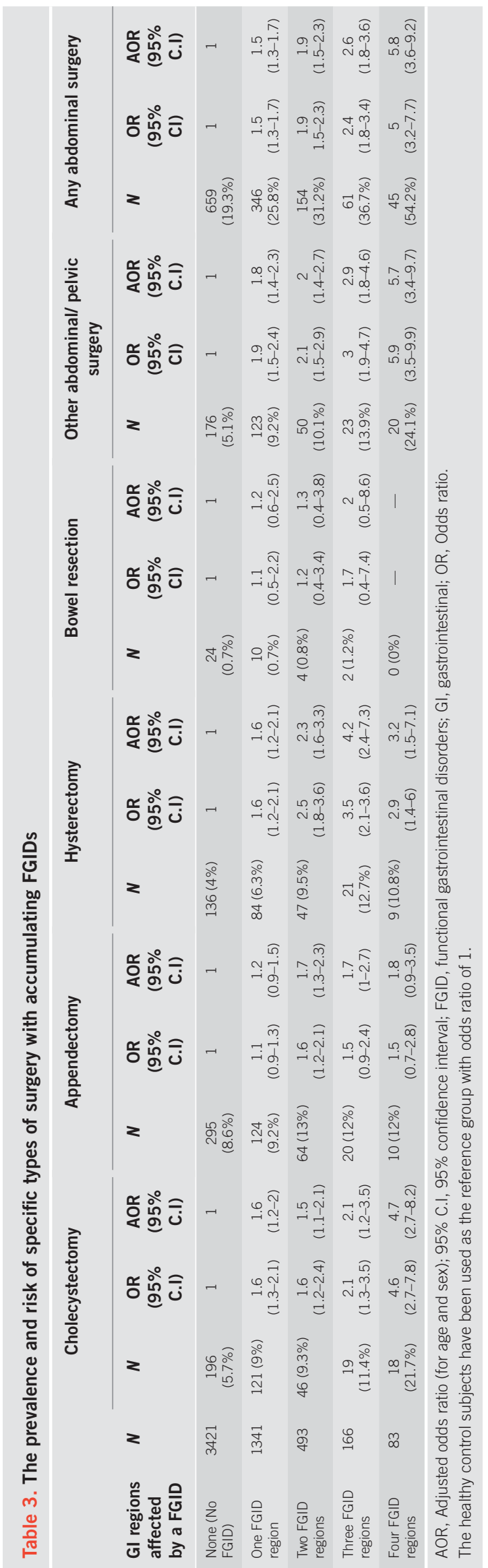

be speculated that their disease etiology is centrally, as opposed to peripherally, mediated. An initial randomized controlled trial found subjects with high levels of somatization and severe IBS to show the greatest degree of benefit from centrally acting desensitizing treatments (such as antidepressants and behavioral therapies) compared with standard treatment modalities (46). However, given the general paucity of data further randomized controlled trials are needed.

With regards to the increased surgical rates we observed in subjects with FGIDs, the reasons are unclear. It is a fi nding that is in agreement with multiple previous studies, and various explanations have been proposed. Th ese include misdiagnosis of FGIDs resulting in a surgical operation or, conversely, FGID symptoms occurring as a consequence of an operation (24-34). For example, recent insights demonstrate that gut visceral hypersensitivity is frequently responsible for the disproportionate pain experienced by patients with suspected endometriosis, in particular those who have normal to minimal laparoscopic fi $\mathrm{n}$ dings a nd $\mathrm{m}$ ay $\mathrm{h}$ ave otherwise gone on to have a hysterectomy (47). On the other hand, a cholecystectomy can lead to bile acid malabsorption mimicking functional diarrhea or diarrhea predominant IBS (48). The se are plausible reasons given that the increased risk of surgery in FGIDs tracked only for hysterectomy and cholecystectomy, but not appendectomy or bowel resection where surgery would generally be predicated on objective indications. Our study did not assess the temporal relationship between fi rst onset of symptoms of FGIDs and surgery, but another plausible hypothesis worthy of consideration, given the associated somatization, is that surgery is being undertaken in co-existing viscerally sensitive somatic sites. For instance, period pains and dyspareunia are commonly reported in IBS patients potentially prompting hysterectomies (49-51). Another somatic symptom is back pain and there is evidence to show a $50 \%$ increase in back surgery for subjects with IBS compared with healthy controls (26). Unfortunately, when subjects have undergone such surgical interventions the resected specimen is generally normal and moreover patients do not report a clinical improvement $(52,53)$.

A key strength of our study is that it is the first one to sample the prevalence and impact of all FGIDs, using the validated new Rome IV diagnostic questionnaire across a large number of individuals in the general adult population in three Englishspeaking countries. In contrast, previous studies have been limited to evaluating the prevalence and eff ect of FGIDs within single communities or focusing on distinct clinical entities such as IBS and functional dyspepsia (3-38). In some countries the Rome III diagnostic questionnaire was modifi ed and translated into other languages without validation $(15,16)$. Hence, we believe that our study provides confi dence in the accuracy and consistent interpretation of the Rome IV questionnaire thereby allowing the results to be generalized.

Nevertheless, our study does have several limitations. First, this data set does not cover the epidemiology of FGIDs globally. At the time the survey was conducted, no professional translations of the new Rome IV diagnostic questionnaire had yet been completed, so we could only survey English-speaking subjects. Th i s r estricted 
our choice of countries to be included and also meant that in Canada, only individuals who were fl $\mathrm{u}$ ent in $\mathrm{E}$ nglish c ould participate, which may have excluded as many as one in fi ve Canadian adults. Generalizability of our fi ndings to non-English speaking populations is unknown at this point, but will be elucidated by the fi ndings of a Global FGID survey with comparable methodology which is now under way.

A second limitation was that despite the overall prevalence of FGIDs being similar across the countries, we noted that people in the USA are more likely to have functional upper GI disorders, and we did not collect information that could explain this difference. Other groups have noted that global prevalence rates of GI disorders vary widely, with the diff erences proposed to occur as a result of genetic, ethnic, cultural, socio-economic, and environmental factors plus the respective healthcare policies within each country $(10-14,54)$. With regards to dyspeptic symptoms specifi $\mathrm{c}$ ally, m odestly a ssociated $\mathrm{r}$ isk $\mathrm{f}$ actors i nclude $\mathrm{s}$ moking, nonsteroidal anti-infl a mmatory $\mathrm{d}$ rug $\mathrm{u}$ sage, a nd Helicobacter pylori status; these factors were not explored in our study (14). Th ird, it has also been shown that over time FGID subtypes do change into others although the overall prevalence remains relatively stable (55). Th is could not be assessed in our study owing to its cross-sectional nature. A fourth limitation to be acknowledged is that despite identifying subjects with common doctordiagnosed organic GI diseases, as well as those who fulfilled criteria for Rome IV FGIDs, we could potentially have missed less common GI conditions. Moreover, we did not have access to medical records and neither could we perform any investigations to confi rm or refute these diagnoses. Previous studies of the Rome III criteria for IBS, functional dyspepsia, and functional bowel disorders in secondary-care settings have shown that they perform modestly when it comes to excluding organic diseases (56-58). However, such data have not been available for the general population and future studies would benefi $t$ from evaluating how the Rome IV performs in excluding organic diseases.

Finally, our study utilized Internet survey methodology, which, despite largely being advantageous compared to tradi-tional postal or telephone surveys, may still introduce several sources of bias. Th ese issues are extensively discussed in the Supplementary Materials. In summary, online surveys are attractive owing to their costeff ectiveness, time convenience, and ability to reach large sample sizes. However, owing to our survey methodology, we were not able to calculate participation rate, as potential subjects in the target demographic categories received e-mail notifi cations that a new health survey was avail-able for them, but it is unknown how many saw this notice or how many responded to it by viewing the consent page. Only those who consented to enter the survey could be counted by our survey method. Furthermore, despite the annual growth of the Internet, between 15 and $20 \%$ of USA and UK households still do not have Internet access (online national govern-ment data). Th ere is also the issue of sample bias, although we addressed this by performing quota-based sampling to gener-ate demographically balanced and population representative samples with regards to age, sex, and education level. Further, we also introduced the survey as one of general health and not GI-related. Finally, data quality checks were incorporated to ensure no missing data and that any inconsistent responders were eliminated from the final analysis. We therefore believe that we undertook necessary measures to counteract potential bias associated with online surveys and provide a representative epidemiological profile.

In conclusion, roughly a third of the general adult population fulfils diagnostic criteria for Rome IV FGIDs. Furthermore, in a third of this subset multiple regions of the GI tract are involved and this more widespread presence of FGID symptoms is associated with increased somatization, poorer QOL, and greater healthcare utilization. These findings have important public health implications as awareness of the epidemiological scale of FGIDs and the detrimental effect on general health and well-being, resulting from the co-existence of FGIDs in multiple GI regions will aid clinical service and research provision planning, and underlines the importance of comprehensive FGID assessment of patients.

\section{ACKNOWLEDGMENTS}

The study was performed in accordance with the STROBE statement. The study was approved by the Institutional Review Board of the University of North Carolina, United States.

\section{CONFLICT OF INTEREST}

Guarantor of the article: Imran Aziz, MBChB, MD. Specific author contributions: OSP and WEW contributed to the study design, questionnaire development, execution of survey, data processing, and manuscript editing. ADS contributed to the study design, questionnaire development, and manuscript editing. HT contributed to interpretation of data and manuscript editing. MS contributed to study design, interpretation of data and manuscript editing. IA analyzed the data and drafted the manuscript. All authors reviewed and approved the final version of the manuscript.

Financial Support: This study was supported by the Rome Foundation, NIDDK (grant RO1 DK31369), the Swedish Medical Research Council (grants 13409, 21691 and 21692), AFA Insurance, an unrestricted grant from Ferring Pharmaceuticals, and by the Faculty of Medicine, University of Gothenburg.

Potential competing interests: MS has received unrestricted research grants from Danone, and Ferring Pharmaceuticals, and served as a Consultant/Advisory Board member for AstraZeneca, Danone, Nestlé, Almirall, Allergan, Albireo, Glycom, and Shire, and as a speaker for Tillotts, Menarini, Takeda, Shire, Allergan, and Almirall. HT has served as Consultant/Advisory Board member for Almirall, Danone, and Shire. OSP has received salary support from a research grants from Takeda Pharmaceuticals and Salix Pharmaceuticals and from a consulting agreement with Ironwood Pharmaceuticals and an educational grant provided by Takeda Pharmaceuticals, and received a speaker honorarium in an educational programe supported by Ironwood Pharmaceuticals and Takeda Pharmaceuticals. WEW received research grants from Takeda, Ironwood, Salix, and the Rome Foundation; served as a consultant to Biomerica USA, Ono Pharmaceuticals and Ferring; and received unrestricted educational grants from Takeda and Ferring. 


\section{Study Highlights}

\section{WHAT IS CURRENT KNOWLEDGE}

Functional gastrointestinal disorders (FGIDs) are currently defined in accordance with the Rome IV diagnostic questionnaire.

Overlapping gastrointestinal symptoms are commonly reported by subjects with FGIDs.

There are no data on the prevalence of Rome IV-diagnosed FGIDs, the magnitude of their overlap and how this overlap impacts general health.

\section{WHAT IS NEW HERE}

Roughly, a third of the general adult population in a large population survey from the USA, Canada, and UK fulfils Rome IV diagnostic criteria for FGIDs, and in a third of those with FGIDs more than one GI region is involved.

There is a direct association between the number of different GI regions with FGIDs and increasing somatization, worse quality of life (QOL), and greater healthcare utilization.

Importantly, presence of multiple FGIDs is associated with greater somatization and worse QOL than is seen in patients with organic GI disease.

\section{REFERENCES}

1. Drossman DA, Hasler WL. Rome IV-functional GI disorders: disorders of gut-brain interaction. Gastroenterology 2016;150:1257-61.

2. Drossman DA. functional gastrointestinal disorders: history, pathophysiology, clinical features and Rome IV. Gastroenterology 2016;150: 1262-79.

3. Chang L. Review article: epidemiology and quality of life in functional gastrointestinal disorders. Aliment Pharmacol Ther 2004;20:31-9.

4. Talley NJ. Functional gastrointestinal disorders as a public health problem. Neurogastroenterol Motil 2008;20:121-9.

5. Drossman DA, Li Z, Andruzzi E et al. U.S. householder survey of functional gastrointestinal disorders. Prevalence, sociodemography, and health impact. Dig Dis Sci 1993;38:1569-80.

6. Locke GR. The epidemiology of functional gastrointestinal disorders in North America. Gastroenterol Clin North Am 1996;25:1-19.

7. Koloski NA, Talley NJ, Boyce PM. Epidemiology and health care seeking in the functional GI disorders: a population-based study. Am J Gastroenterol 2002;97:2290-9.

8. Locke GR, Zinsmeister AR, Fett SL et al. Overlap of gastrointestinal symptom complexes in a US community. Neurogastroenterol Motil 2005;17:29-34

9. Boyce PM, Talley NJ, Burke C et al. Epidemiology of the functional gastrointestinal disorders diagnosed according to Rome II criteria: an Australian population-based study. Intern Med J 2006;36:28-36

10. Ford AC, Marwaha A, Lim A et al. Systematic review and meta-analysis of the prevalence of irritable bowel syndrome in individuals with dyspepsia. Clin Gastroenterol Hepatol 2010;8:401-9.

11. Suares NC, Ford AC. Prevalence of, and risk factors for, chronic idiopathic constipation in the community: systematic review and meta-analysis. Am J Gastroenterol 2011;106:1582-91.quiz 1581, 1592

12. Lovell RM, Ford AC. Global prevalence of and risk factors for irritable bowel syndrome: a meta-analysis. Clin Gastroenterol Hepatol 2012;10: 712-721.e714.

13. Lovell RM, Ford AC. Prevalence of gastro-esophageal reflux-type symptoms in individuals with irritable bowel syndrome in the community: a metaanalysis. Am J Gastroenterol 2012;107:1793-801.quiz 1802.

14. Ford AC, Marwaha A, Sood R et al. Global prevalence of, and risk factors for, uninvestigated dyspepsia: a meta-analysis. Gut 2015;64:1049-57.

15. Park JM, Choi MG, Cho YK et al. Functional gastrointestinal disorders diagnosed by Rome III questionnaire in Korea. J Neurogastroenterol Motil 2011;17:279-86
16. Wu W, Guo X, Yang Y et al. The prevalence of functional gastrointestinal disorders in the chinese air force population. Gastroenterol Res Pract 2013;2013:497585.

17. Qumseya BJ, Tayem Y, Almansa C et al. Irritable bowel syndrome in middle-aged and elderly Palestinians: its prevalence and effect of location of residence. Am J Gastroenterol 2014;109:723-39.

18. Riedl A, Schmidtmann M, Stengel A et al. Somatic comorbidities of irritable bowel syndrome: a systematic analysis. J Psychosom Res 2008;64: $573-82$.

19. Patel P, Ford AC. Irritable bowel syndrome and somatization. Neurogastroenterol Motil 2015;27:740.

20. Patel P, Bercik P, Morgan DG et al. Irritable bowel syndrome is significantly associated with somatisation in 840 patients, which may drive bloating. Aliment Pharmacol Ther 2015;41:449-58.

21. North CS, Downs D, Clouse RE et al. The presentation of irritable bowel syndrome in the context of somatization disorder. Clin Gastroenterol Hepatol 2004;2:787-95.

22. Koloski NA, Talley NJ, Boyce PM. The impact of functional gastrointestinal disorders on quality of life. Am J Gastroenterol 2000;95:67-71.

23. Ford AC, Forman D, Bailey AG et al. Initial poor quality of life and new onset of dyspepsia: results from a longitudinal 10-year follow-up study. Gut 2007;56:321-7.

24. McNally MA, Locke GR, Zinsmeister AR et al. Biliary events and an increased risk of new onset irritable bowel syndrome: a population-based cohort study. Aliment Pharmacol Ther 2008;28:334-43.

25. Longstreth GF, Preskill DB, Youkeles L. Irritable bowel syndrome in women having diagnostic laparoscopy or hysterectomy. Relation to gynecologic features and outcome. Dig Dis Sci 1990;35:1285-90.

26. Longstreth GF, Yao JF. Irritable bowel syndrome and surgery: a multivariable analysis. Gastroenterology 2004;126:1665-73.

27. Thistle JL, Longstreth GF, Romero Y et al. Factors that predict relief from upper abdominal pain after cholecystectomy. Clin Gastroenterol Hepatol 2011;9:891-6.

28. Kennedy TM, Jones RH. Epidemiology of cholecystectomy and irritable bowel syndrome in a UK population. Br J Surg 2000;87:1658-63.

29. Kennedy TM, Jones RH. The epidemiology of hysterectomy and irritable bowel syndrome in a UK population. Int J Clin Pract 2000;54:647-50.

30. Burns DG. The risk of abdominal surgery in irritable bowel syndrome. S Afr Med J 1986;70:91.

31. Sperber AD, Morris CB, Greemberg L et al. Development of abdominal pain and IBS following gynecological surgery: a prospective, controlled study. Gastroenterology 2008;134:75-84

32. Lu CL, Liu CC, Fuh JL et al. Irritable bowel syndrome and negative appendectomy: a prospective multivariable investigation. Gut 2007;56: 655-60.

33. Kirk G, Kennedy R, McKie L et al. Preoperative symptoms of irritable bowel syndrome predict poor outcome after laparoscopic cholecystectomy. Surg Endosc 2011;25:3379-84.

34. Hasler WL, Schoenfeld P. Systematic review: abdominal and pelvic surgery in patients with irritable bowel syndrome. Aliment Pharmacol Ther 2003;17:997-1005.

35. Kaji M, Fujiwara Y, Shiba M et al. Prevalence of overlaps between GERD, FD and IBS and impact on health-related quality of life. J Gastroenterol Hepatol 2010;25:1151-6.

36. Pinto-Sanchez MI, Ford AC, Avila CA et al. Anxiety and depression increase in a stepwise manner in parallel with multiple FGIDs and symptom severity and frequency. Am J Gastroenterol 2015;110:1038-48.

37. Vakil N, Stelwagon M, Shea EP et al. Symptom burden and consulting behavior in patients with overlapping functional disorders in the US population. United European Gastroenterol J 2016;4:413-22.

38. Choung RS, Richard Locke G, Schleck CD et al. Multiple functional gastrointestinal disorders linked to gastroesophageal reflux and somatization: a population-based study. Neurogastroenterol Motil 2017;29:

39. Palsson OS, Whitehead WE, van Tilburg MA et al. Rome IV diagnostic questionnaires and tables for investigators and clinicians. Gastroenterology 2016;150:1481-91.

40. Spiller RC, Humes DJ, Campbell E et al. The Patient Health Questionnaire 12 Somatic Symptom scale as a predictor of symptom severity and consulting behaviour in patients with irritable bowel syndrome and symptomatic diverticular disease. Aliment Pharmacol Ther 2010;32:811-20.

41. Kroenke K, Spitzer RL, Williams JB. The PHQ-15: validity of a new measure for evaluating the severity of somatic symptoms. Psychosom Med 2002;64:258-66. 
42. Ware JE, Kosinski M, Dewey JE et al. How to Score and Interpret SingleItem Health Status Measures: A Manual for Users of the SF-8 Health Survey. Lincoln RI:Quality Metric, 2001.

43. Cohen J. Statistical Power Analysis For The Behavioral Sciences. Routledge, 1988.

44. Everhart JE, Ruhl CE. Burden of digestive diseases in the United States part I: overall and upper gastrointestinal diseases. Gastroenterology 2009;136:376-86.

45. Ringström G, Abrahamsson $\mathrm{H}$, Strid $\mathrm{H}$ et al. Why do subjects with irritable bowel syndrome seek health care for their symptoms? Scand J Gastroenterol 2007;42:1194-203.

46. Creed F, Tomenson B, Guthrie E et al. The relationship between somatisation and outcome in patients with severe irritable bowel syndrome. J Psychosom Res 2008;64:613-20.

47. Issa B, Onon TS, Agrawal A et al. Visceral hypersensitivity in endometriosis: a new target for treatment? Gut 2012;61:367-72.

48. Mottacki N, Simrén M, Bajor A. Review article: bile acid diarrhoea-pathogenesis, diagnosis and management. Aliment Pharmacol Ther 2016;43:884-98

49. Bass C, Murphy M. Somatisation disorder in a British teaching hospital. Br J Clin Pract 1991;45:237-44.

50. Longstreth GF. Irritable bowel syndrome and chronic pelvic pain. Obstet Gynecol Surv 1994;49:505-7.
51. Choung RS, Herrick LM, Locke GR et al. Irritable bowel syndrome and chronic pelvic pain: a population-based study. J Clin Gastroenterol 2010;44:696-701

52. Talley NJ. Unnecessary abdominal and back surgery in irritable bowel syndrome: time to stem the flood now? Gastroenterology 2004;126:1899-903.

53. Longstreth GF. Avoiding unnecessary surgery in irritable bowel syndrome. Gut 2007;56:608-10.

54. Mahadeva S, Ford AC. Clinical and epidemiological differences in functional dyspepsia between the East and the West. Neurogastroenterol Motil 2016;28:167-74.

55. Halder SL, Locke GR, Schleck CD et al. Natural history of functional gastrointestinal disorders: a 12-year longitudinal population-based study. Gastroenterology 2007;133:799-807

56. Ford AC, Bercik P, Morgan DG et al. Validation of the Rome III criteria for the diagnosis of irritable bowel syndrome in secondary care. Gastroenterology 2013;145:1262-1270.e1261.

57. Ford AC, Bercik P, Morgan DG et al. The Rome III criteria for the diagnosis of functional dyspepsia in secondary care are not superior to previous definitions. Gastroenterology 2014;146:932-40.

58. Ford AC, Bercik P, Morgan DG et al. Characteristics of functional bowel disorder patients: a cross-sectional survey using the Rome III criteria. Aliment Pharmacol Ther 2014;39:312-21. 